\title{
LOCAL PROPERTIES OF MAPS OF THE BALL
}

\author{
YAKAR KANNAI
}

Received 25 January 2002

Let $f$ be an essential map of $S^{n-1}$ into itself (i.e., $f$ is not homotopic to a constant mapping) admitting an extension mapping the closed unit ball $\bar{B}^{n}$ into $\mathbb{R}^{n}$. Then, for every interior point $y$ of $B^{n}$, there exists a point $x$ in $f^{-1}(y)$ such that the image of no neighborhood of $x$ is contained in a coordinate half space with $y$ on its boundary. Under additional conditions, the image of a neighborhood of $x$ covers a neighborhood of $y$. Differential versions are valid for quasianalytic functions. These results are motivated by game-theoretic considerations.

\section{Introduction}

Let $\Omega$ be an open subset of $\mathbb{R}^{n}$, and let $f$ be a real continuous function defined in $\Omega$. We say that $f$ is one-sided at a point $\bar{x} \in \Omega$, if there exists a neighborhood $U$ of $\bar{x}$, such that either $f(x) \geq f(\bar{x})$ or $f(x) \leq f(\bar{x})$ for $x \in U$, but $f$ is not constant on $U$. Otherwise (i.e., if either $f(x)=f(\bar{x})$ near $\bar{x}$ or $f(x)-f(\bar{x})$ assumes both positive and negative values at every neighborhood of $\bar{x}) f$ is multisided at $\bar{x}$. If $f$ is a continuous function $f: \Omega \rightarrow \mathbb{R}^{n}$, then $f$ is multisided at $\bar{x}$ if every component $f_{i}(x)$ is multisided at $\bar{x}$.

Theorem 1.1. Let $B=B^{n}$ denote the open unit ball of $\mathbb{R}^{n}$. Let $f$ be a continuous map of $\bar{B}$ into $\mathbb{R}^{n}$ such that $f: \partial B\left(=S^{n-1}\right) \rightarrow \partial B$, and $\left.f\right|_{\partial B}$ is an essential map. Then, for every $y \in B$ there exists $\bar{x} \in f^{-1}(y)$ such that $f$ is multisided at $\bar{x}$.

Additional assumptions on $f$ imply that there exist points in $f^{-1}(y)$ whose neighborhoods are mapped into neighborhoods of $y$. (Additional assumptions are needed, see Examples 3.1 and 3.2.)

Theorem 1.2. Let $B$ and $f$ be as in Theorem 1.1. Let $y \in B$, such that the inverse image of $y$ is zero dimensional at every point in the closure of the set 
$\left\{x: x \in f^{-1}(y), f\right.$ is multisided at $\left.x\right\}$. Then there exists $\bar{x} \in f^{-1}(y)$ such that for every open neighborhood $U$ of $\bar{x}, f(U)$ contains an open neighborhood of $y$.

There exists differential (actually, quasianalytic) versions of the preceding concepts. Let $\mathbf{M}=M_{j}$ be a logarithmically convex sequence of positive numbers. Let $V$ be an open subset of $\mathbb{R}^{k}$, where $k$ is arbitrary. The class $C_{\mathbf{M}}(V)$ consists of those functions $f$ in $C^{\infty}(V)$ such that for every compact $K \subset V$ there exists constants $A$ and $B$ such that for every multi-index $\alpha$, the inequality

$$
\left|\partial^{\alpha} f(x)\right| \leq A B^{|\alpha|} M_{|\alpha|}
$$

holds whenever $x \in K$. The well-known Denjoy-Carleman theorem [3] states that the class $C_{\mathbf{M}}(V)$ is quasianalytic if and only if the series $\sum_{j=1}^{\infty} M_{j-1} / M_{j}$ diverges. Following [2], we may consider $C_{M}$ manifolds, in particular we consider $C_{M}$ curves, that is, curves which possess a parametric representation $x(t)=$ $\left(x_{1}(t), \ldots, x_{n}(t)\right)$, such that the functions $x_{i}(t)$ are in the class $C_{M}$, for all $1 \leq i \leq n$.

A scalar function $f \in C_{\mathbf{M}}(\Omega)$ is $C_{\mathbf{M}}$ one-sided at a point $\bar{x} \in \Omega$ if

(i) there exists a $C_{\mathbf{M}}$ curve $x(t)$ such that $x(0)=\bar{x}$, and $f(x(t))$ is not locally constant at $t=0$,

(ii) for every $C_{\mathbf{M}}$ curve $x(t)$ such that $x(0)=\bar{x}$, either

(1) the first nonvanishing derivative of $f(x(t))$ (with respect to $t$ at $t=0)$ is of even order and is positive or

(2) the first nonvanishing derivative of $f(x(t))$ is of even order and is negative or

(3) $f(x(t)) \equiv f(\bar{x})$.

If $f \rightarrow \mathbb{R}^{n}, f \in C_{\mathbf{M}}$, and the function $f_{i}(x)$ is $C_{\mathbf{M}}$ one-sided at $\bar{x}$ for no $1 \leq i \leq n$ is, we say that $f$ is $C_{\mathbf{M}}$ multisided at $\bar{x}$.

The relation between ordinary one-sidedness and $C_{M}$ one-sidedness is spelled out in the following proposition.

Proposition 1.3. Let $f$ be a scalar function, $f \in C_{\mathbf{M}}(\Omega)$. Then, $f$ is one-sided at $\bar{x} \in \Omega$ if and only if $f$ is $C_{\mathbf{M}}$ one-sided at $\bar{x}$.

The proof of Proposition 1.3 depends on the desingularization results of Bierstone and Milman [2]. The proposition implies immediately the following version of Theorem 1.1.

Theorem 1.4. Let $B$ and $f$ be as in Theorem 1.1, and let $f \in C_{\mathbf{M}}(B)$. Then there exists $\bar{x} \in f^{-1}(y)$ such that $f$ is $C_{\mathrm{M}}$ multisided at $\bar{x}$.

Note that the boundary behavior of $f$ along with quasianalyticity imply that no component $f_{i}$ of $f$ is locally constant at any point. As seen in Example 3.3, we do not have a $C_{M}$ strengthening of Theorem 1.2.

Observe that Proposition 1.3 and Theorem 1.4 are valid if, instead of assuming that $M$ is a Denjoy-Carleman class, we assume that $M=C(\Omega)$, where the collection of algebras $C(U)$ ( $U$ runs over all open subsets of $\mathbb{R}^{m}, m$ arbitrary) 
satisfies conditions (3.1), (3.2), (3.3), (3.4), (3.5), and (3.6) of [2]. As a matter of fact, only these assumptions are needed for the validity of the desingularization results in [2].

The motivation for the study of multisidedness comes from recent results on set-valued maps of the closed simplex $\Delta$ (see [4]), results which have gametheoretic applications. For example, it is shown that under certain conditions, if $F$ is a set-valued map from $\Delta$ to the convex subsets of $\Delta$, then for every $y \in$ $\operatorname{int}(\Delta)$, there exists $x$, such that $y$ is in the relative interior of $F(x)$ (see [4, Theorem 1]). If in addition, the closure of the set $\{x: y \in \operatorname{relint}(F(x))\}$ is zero dimensional, then there exists $\bar{x} \in \operatorname{int}(\Delta)$ such that $y \in \operatorname{int}(F(\bar{x}))$ (see $[4$, Theorem $2])$. The purpose of this paper is to find a single-valued analog of the results of [4]. The proofs of Theorems 1.1 and 1.2 here are similar in spirit to the proofs of Theorems 1 and 2 in [4], respectively. In [4], the maps are not deformed continuously (as in the present paper); instead, certain neighborhoods of the origin are removed from the values of the (set-valued) maps. The weaker conclusion in Theorem 1.1 as well as the slightly weaker zero-dimensionality assumption in Theorem 2 of [4] are compensated by a certain finiteness assumption made in [4].

Note that, Theorems 1.1 and 1.4 continue to hold, if we replace the standard base of $\mathbb{R}^{n}$ by any other basis $\mathbf{v}_{1}, \ldots, \mathbf{v}_{n}$, write $f$ as $\sum_{i=1}^{n} \hat{f}_{i}(x) \mathbf{v}_{i}$, and define onesidedness and multisidedness using the functions $\hat{f}_{i}$. (This was pointed out by I. Yomdin (personal communication).)

Note that, unlike the situation in Sard's theorem, Theorem 1.1 applies to all points $y \in B$.

\section{Proofs}

Let $f$ satisfy the assumptions of Theorem 1.1, and let $y$ be an arbitrary point of $B$. We may assume, without loss of generality (to simplify notation a bit), that $y=0$. Let $\bar{x}$ be a point in $D_{i}=f_{i}^{-1}(0) \cap B$. Let $C_{i}^{+}\left(C_{i}^{-}\right)$denote the set of points $\bar{x} \in D_{i}$ for which there exists a neighborhood $U$ of $\bar{x}$ in which $f_{i}(x) \geq 0\left(f_{i}(x) \leq\right.$ 0 ), but $f_{i}(x)$ is not a constant in $U$ (i.e., $f_{i}(x)$ does not vanish identically in $U$ ). Set $N_{i}=f_{i}^{-1}(-\infty, 0)$ and $P_{i}=f_{i}^{-1}(0, \infty)$. For any set $A$, let $\rho(x, A)$ denote the distance of $x$ from $A$ ( $\left.=\inf _{y \in A}|x-y|\right)$. We now deform the map $f$ as follows: for every $t \geq 0$, set

$$
f_{t}(x)=\left(f_{1}(x, t), \ldots, f_{n}(x, t)\right)
$$

where

$$
f_{i}(x, t)=f_{i}(x)+t \rho(x, \partial B)\left[\rho\left(x, N_{i}\right)-\rho\left(x, P_{i}\right)\right]
$$

for $1 \leq i \leq n$. 
Observe that $f_{t}(x) \equiv f(x)$, if $x \in \partial B$. If $x \in C_{i}^{+} \cup P_{i}$, then $\rho(x, \partial B)>0$ and $\rho\left(x, P_{i}\right)=0$. Moreover, $f_{i}$ is nonnegative in a full neighborhood of $x$. Hence, $\rho\left(x, N_{i}\right)>0$. Thus, $f_{i}(x, t)>f_{i}(x) \geq 0$ if $t>0$. Similarly, if $x \in C_{i}^{-} \cup N_{i}$, then $f_{i}(x, t)$ $<0$ (for positive $t$ ). It follows that $f_{t}(x)=0$ is possible for $t>0$ only if $x \in$ $f^{-1}(0) \backslash \bigcup_{i=1}^{n}\left(C_{i}^{+} \cup C_{i}^{-}\right)$. But the restriction of $f_{t}(x)$ to the boundary $\partial B$ is essential for positive $t$ as well, so that the set $f_{t}^{-1}(0)$ is nonempty. Hence, there exists $\bar{x} \in f^{-1}(0) \backslash \bigcup_{i=1}^{n}\left(C_{i}^{+} \cup C_{i}^{-}\right)$. This means that for every $1 \leq i \leq n, f_{i}(\bar{x})=0$, but $\bar{x}$ is neither in $C_{i}^{+}$nor in $C_{i}^{-}$. This means that either there exists a neighborhood of $\bar{x}$, where $f_{i}$ vanishes identically, or in every neighborhood of $\bar{x} f_{i}$ assumes both positive and negative values. Thus, $f$ is multisided at $\bar{x}$, proving Theorem 1.1.

In proving Theorem 1.2, we continue to use the notations introduced in the proof of Theorem 1.1. Assume, without loss of generality, that the set $f^{-1}(0)$ is zero dimensional at each point of the closure of the set $F=\left\{x: x \in f^{-1}(0), f\right.$ is multisided at $x\}$. Note that $F=f^{-1}(0) \backslash \bigcup_{i=1}^{n}\left(C_{i}^{+} \cup C_{i}^{-}\right)$, and that the deformations defined by (2.1) and (2.2) eliminate all zeros of $f$ outside $F$ without introducing new ones. It is possible, however, that $f_{t}(x)$ will no longer vanish (for $t>0$ ) on all of $F$. We modify the deformation by setting

$$
g_{t}(x)=\left(g_{1}(x, t), \ldots, g_{n}(x, t)\right)
$$

where

$$
g_{i}(x, t)=f_{i}(x)+t \rho(x, \partial B) \rho(x, F)\left[\rho\left(x, N_{i}\right)-\rho\left(x, P_{i}\right)\right]
$$

for $1 \leq i \leq n$.

It is easily seen that $g_{t}(x) \neq 0$ if $t>0$ and $x \notin \bar{F}$. The assumptions on boundary behavior imply that for all $t \geq 0$, we have

$$
d\left(g_{t}, B, 0\right) \neq 0 \text {. }
$$

(We use standard notation for degree.)

By assumption, the set $f^{-1}(0)$ is zero dimensional at every point of the compact set $\bar{F}$. This implies that for every $\epsilon>0$, the set $\bar{F}$ may be covered by a finite number of disjoint open sets whose diameter is less than $\epsilon$, and whose boundaries do not meet $f^{-1}(0)$. Let $\left\{D_{i, m}\right\}_{i=1}^{P_{m}}$ denote such a collection of sets with $\operatorname{diam}\left(D_{i, m}\right)<1 / m, \bar{F} \subseteq \bigcup_{i=1}^{P_{m}} D_{i, m}, D_{i, m} \cap D_{j, m}=\varnothing$ for $i \neq j$, and $\partial D_{i, m} \cap$ $f^{-1}(0)=\varnothing$. By construction, if $t \geq 0$, then $g_{t}(x) \neq 0$ for all $x \in \partial D_{i, m}, 1 \leq i \leq P_{m}$. Hence, $d\left(g_{t}, D_{i, m}, 0\right)$ is well defined for all $t \geq 0$ and

$$
\sum_{i=1}^{P_{m}} d\left(g_{t}, D_{i, m}, 0\right)=d\left(g_{t}, B, 0\right)
$$


for all positive $t$. It follows from (2.5) and (2.6) that there exists $i_{0}=i_{0}(\mathrm{~m})$ such that $d\left(g_{1 / m}, D_{i_{0}(m), m}, 0\right) \neq 0$. By compactness, there exist a point $\bar{x} \in \bar{F}$ and a sequence $D_{i_{0}(m), m}$ of neighborhoods (with $D_{i_{0}(m), m} \cap \bar{F}$ compact) such that $\bar{x}=\bigcap_{m=1}^{\infty} D_{i_{0}(m), m}$. By construction, the degree $d\left(g_{t}, D_{i_{0}(m), m}, 0\right)$ is independent of $t \geq 0$. For every open neighborhood $U$ of $\bar{x}$, there exists a number $M$ such that the ball around $\bar{x}$ with radius $1 / M$ is contained in $U$, so that $D_{i_{0}(m), m} \subset U$ if $m>2 M$. It follows that $d\left(f, D_{i_{0}(m), m}, 0\right)=d\left(g_{1 / m}, D_{i_{0}(m), m}, 0\right) \neq 0$. Hence, $f(U)$ contains a full neighborhood of 0 and Theorem 1.2 follows.

The proof of Proposition 1.3 relies on the results of [2]. We may assume, without loss of generality, that $\bar{x}=0$ and $f(0)=0$. Let $h(x)$ be a scalar $C_{\mathbf{M}}$ function in a neighborhood $U$ of the origin, with $h(0)=0$ such that $h$ does not vanish identically in $U$ and $h(x) \geq 0$ in $U$. Let $x(t)$ be an arbitrary $C_{\mathbf{M}}$ curve in $U$ such that $x(0)=0$. If there exists a sequence $t_{n} \rightarrow 0, t_{n} \neq 0$, such that $h\left(x\left(t_{n}\right)\right)=0$ for all $n$, then the quasianalyticity of $h$ and $x$ implies that $h(x(t))$ vanishes identically. Otherwise, the function $h(x(t))$ is strictly positive for small $t \neq 0$. Not all derivative of $h(x(t))$, with respect to $t$, vanish at $t=0$; the first nonzero one must be of even order and positive.

It remains to show that, there exist $C_{\mathbf{M}}$ curves $x(t)$ such that $h(x(t))$ does not vanish identically. This follows from the "curve selection lemma." We prove the existence of such curves following Atiyah (see [1, page 147]) and BierstoneMilman [2], and noting that there exists an $n$-dimensional $C_{M}$ manifold $U^{\prime}$ and a proper $C_{\mathbf{M}}$ map $\phi$ from $U^{\prime}$ onto a neighborhood of the origin (contained in $U)$ such that $\phi^{-1}$ is one to one on $\{x: h(x) \neq 0\}$, and such that $h \circ \phi$ is locally a monomial times an invertible factor (in suitable coordinates). In particular, we may choose a (connected) neighborhood $W$ in $U^{\prime}$ and $C_{\mathbf{M}}$ coordinates $w_{1}, \ldots, w_{n}$ in $W$ such that $\phi(0)=0$ and

$$
h(\phi(w))=g(w) \prod_{i=1}^{n} w_{i}^{k_{i}}
$$

for $w \in W$, where the $C_{\mathrm{M}}$ function $g$ never vanishes in $W$, and $k_{i}$ is a nonnegative integer for $1 \leq i \leq n$. By assumption, $g>0$ in $W$ and the numbers $k_{i}$ are even. Define a $C_{\mathbf{M}}$ curve by $x(t)=\phi(t, \ldots, t)$. Then,

$$
h(x(t))=g(t, \ldots, t) t^{\sum_{i=1}^{n} k_{i}} .
$$

Hence $h$ is $C_{M}$ one-sided at 0 .

Let $h$ be $C_{\mathbf{M}}$ one-sided such that for every $C_{\mathbf{M}}$ curve $x(t)$ with $x(0)=0$, either the first nonvanishing derivative of $h(x(t))$ is positive and of even order, or $h(x(t))=0$. We will show that $h$ is one-sided. If $h(x)$ assumes negative values in every neighborhood of 0 , then either (i) $g(0)>0$ and the numbers $k_{i}$ are not all even, or (ii) $g(0)<0$ and all the numbers $k_{i}$ are even. In case (i), suppose that $k_{1}$ is odd. Considering the $C_{M}$ curve $x(t)=\phi(t, 0, \ldots, 0)$, we see that $h$ is not 
$C_{M}$ one-sided. In case (ii), consider the curve $x(t)=\phi(t, \ldots, t)$. Then (2.8) holds with $g(0)<0$, contradicting the assumption. It follows that there exists a neighborhood of 0 , where $h(x)$ is nonnegative. By assumption, $h(x)$ is not identically equal to zero. Hence $h$ is one-sided. The case where the first nonvanishing derivative of $h(x(t))$ is negative, is similar.

Remark 2.1. If $f \in C_{\mathrm{M}}$ is a vector-valued function such that all components $f_{i}$ are one-sided, then there exists a $C_{M}$ curve passing through $\bar{x}$, such that for each $1 \leq i \leq n$ not all derivatives of $f_{i}(x(t))$ vanish at $t=0$. (Set $h=\prod_{i=1}^{n} f_{i}$.)

\section{Examples}

Example 3.1. The following function $f$ is multisided at the origin and no $f_{i}$ is locally constant, but no neighborhood of the origin is mapped onto a full neighborhood of the origin. Set $f_{1}=x_{1}, f_{2}=x_{1} x_{2}$. If $\left(y_{1}, y_{2}\right)=\left(f_{1}\left(x_{1}, x_{2}\right), f_{2}\left(x_{1}, x_{2}\right)\right)$ and $y_{1}=0$, then $y_{2}=0$, so that no point $\left(0, y_{2}\right)$ with $y_{2} \neq 0$ is in the image of $f$. However, every other line through the origin has points of the image of $f$ on both sides, arbitrarily close to the origin.

Example 3.2. The assumptions of Theorem 1.1 do not imply the conclusion of Theorem 1.2. This can be seen by considering the simple one-dimensional function $f(x)=0$ for $|x|<1 / 2, f(x)=2 x-1$ for $1 / 2 \leq x \leq 1$, and $f(x)=2 x+1$ for $-1 \leq x \leq-1 / 2$. Then, $f$ is multisided at $|x|<1 / 2-$ a one-dimensional set, and the conclusion of Theorem 1.2 fails for $y=0$.

Example 3.3. The inverse image of a point may consist of a closed analytic curve even if $f$ is analytic and the assumptions of Theorem 1.2 are satisfied. Let $1 / 2>$ $\delta>0$ and set

$$
f_{1}=\frac{x_{1}\left[\left(\delta-x_{1}\right)^{2}+x_{2}^{2}-\delta^{2}\right]^{2}}{\left(1-2 \delta x_{1}\right)^{2}}, \quad f_{2}=\frac{x_{2}\left[\left(\delta-x_{1}\right)^{2}+x_{2}^{2}-\delta^{2}\right]^{2}}{\left(1-2 \delta x_{1}\right)^{2}}
$$

Then $f^{-1}(0)$ is a circle of radius $\delta$ centered at $(\delta, 0)$. The function is one-sided at every point of the circle except for the origin. The conclusion of Theorem 1.2 holds at the origin. Nevertheless, there exists a curve through the origin on which both $f_{1}$ and $f_{2}$ vanish. Hence, Theorem 1.2 cannot be strengthened (even in the analytic case) so as to assert that $f$ has a nonvanishing derivative (of a sufficiently high order) on every analytic curve through $\bar{x}$.

\section{Acknowledgments}

The work on this paper started at Weizmann and was completed while the author was visiting the University of Minnesota. The author is indebted to E. Bierstone and P. Milman for letting their unpublished work available to him, and to I. Yomdin for useful discussions. 


\section{References}

[1] M. F. Atiyah, Resolution of singularities and division of distributions, Comm. Pure Appl. Math. 23 (1970), 145-150.

[2] E. Bierstone and P. D. Milman, Resolution of singularities in Denjoy-Carleman classes, http://arxiv.org/abs/math.CV/0108204.

[3] L. Hörmander, The Analysis of Linear Partial Differential Operators. I. Distribution Theory and Fourier Analysis, Grundlehren der Mathematischen Wissenschaften, vol. 256, Springer-Verlag, Berlin, 1983.

[4] Y. Kannai and M. H. Wooders, A further extension of the KKMS theorem, Math. Oper. Res. 25 (2000), no. 4, 539-551.

Yakar Kannai: Department of Mathematics, Weizmann Institute of Science, Rehovot, Israel

E-mail address: kannai@wisdom.weizmann.ac.il 


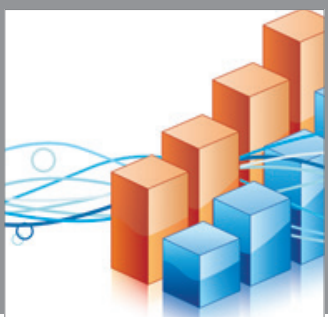

Advances in

Operations Research

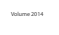

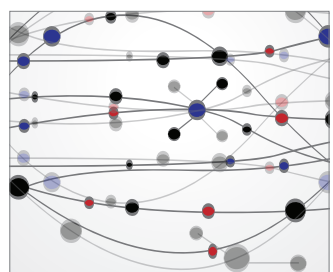

\section{The Scientific} World Journal
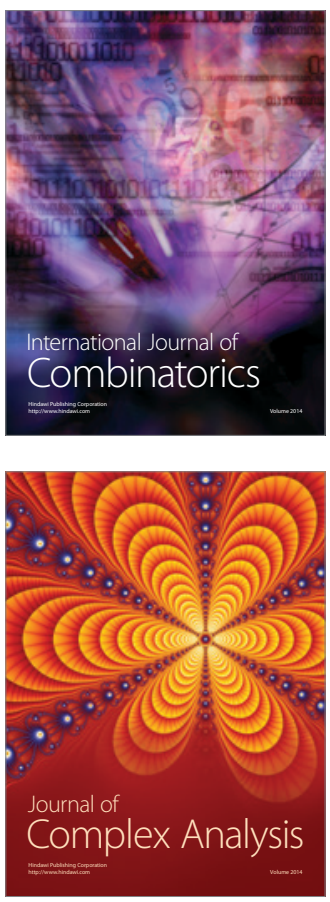

International Journal of

Mathematics and

Mathematical

Sciences
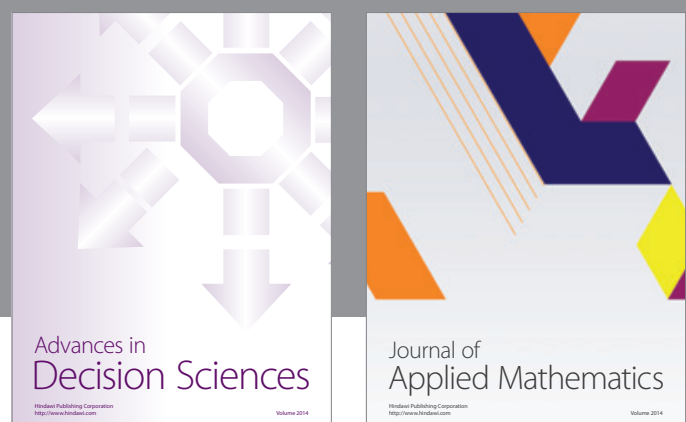

Journal of

Applied Mathematics
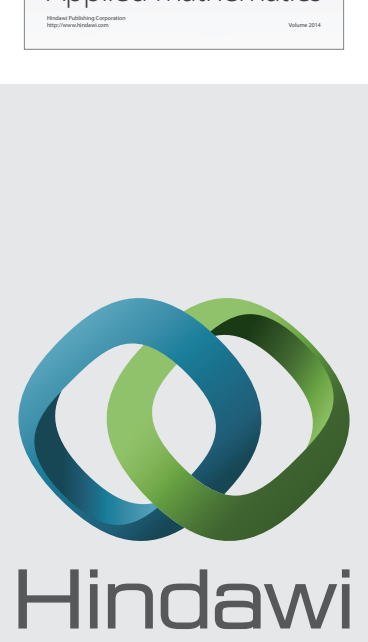

Submit your manuscripts at http://www.hindawi.com
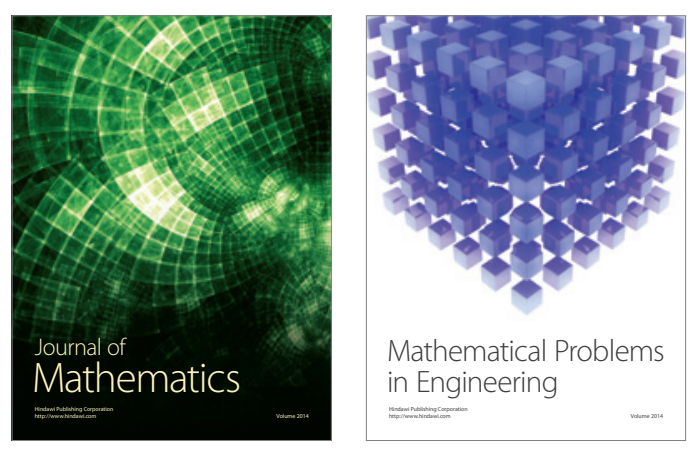

Mathematical Problems in Engineering
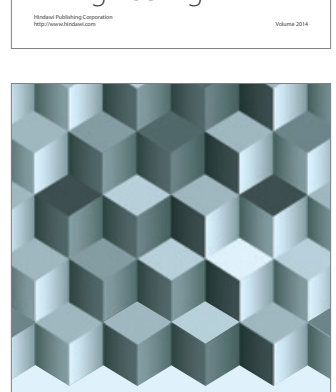

Journal of

Function Spaces
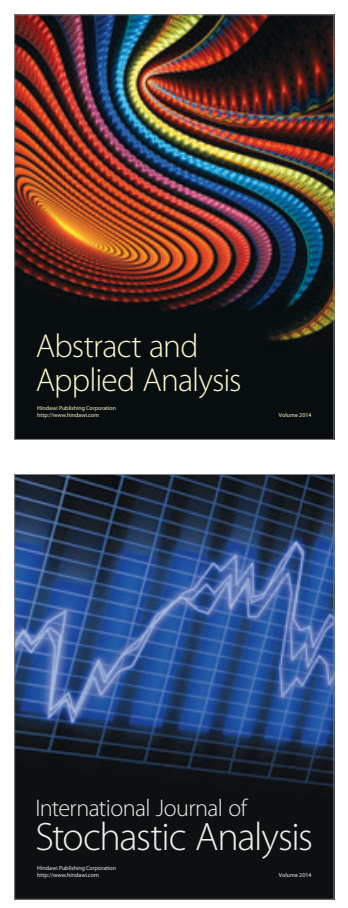

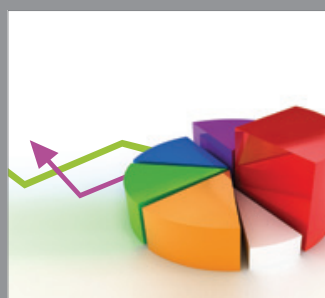

ournal of

Probability and Statistics

Promensencen
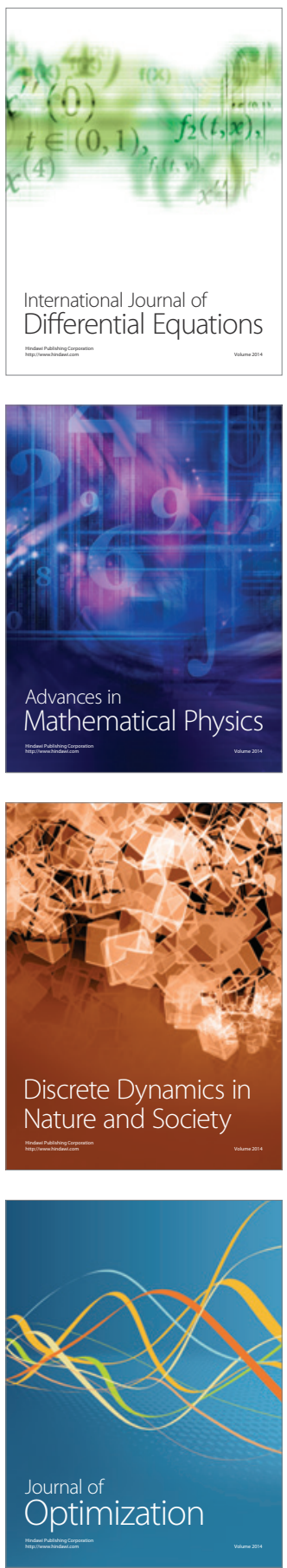